Revue des patrimoines

16 | 2011

Le patrimoine militaire et la question urbaine

\title{
Bunker culturel : la régénération du patrimoine militaire urbain à Saint-Nazaire
}

Renzo Lecardane et Zeila Tesoriere

\section{(2) OpenEdition}

Journals

Édition électronique

URL : http://journals.openedition.org/insitu/779

DOI : $10.4000 /$ insitu. 779

ISSN : 1630-7305

Éditeur

Ministère de la Culture

Référence électronique

Renzo Lecardane et Zeila Tesoriere, «Bunker culturel : la régénération du patrimoine militaire urbain à Saint-Nazaire », In Situ [En ligne], 16 | 2011, mis en ligne le 29 juin 2011, consulté le 10 décembre 2020. URL : http://journals.openedition.org/insitu/779 ; DOI : https://doi.org/10.4000/insitu.779

Ce document a été généré automatiquement le 10 décembre 2020.

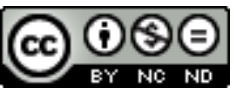

In Situ Revues des patrimoines est mis à disposition selon les termes de la licence Creative Commons Attribution - Pas d'Utilisation Commerciale - Pas de Modification 4.0 International. 


\title{
Bunker culturel : la régénération du patrimoine militaire urbain à Saint- Nazaire
}

\author{
Renzo Lecardane et Zeila Tesoriere
}

1 Au cours des deux dernières décennies, Saint-Nazaire a relancé son développement sans se référer nécessairement aux modèles des métropoles européennes. Concentrées autour de la zone portuaire, près de la base sous-marine allemande, les nouvelles opérations urbaines ont contribué à définir un important projet touristique et culturel. La spécificité symbolique et la valorisation du patrimoine militaire ont permis à Saint-Nazaire de démontrer que l'identité d'une ville pouvait activer sa croissance.

2 À l'instar des premières opérations de waterfront nord-américaines, un grand nombre de réalisations françaises adopte l'approche de l'urban entertainment center, dont la vocation principale est de concentrer dans d'anciennes friches patrimonialisées des activités de commerces et de services récréatifs et culturels ${ }^{1}$.

3 Enjeu identitaire, l'eau constitue un élément de mémoire du lieu qu'il est question aujourd'hui à la fois de révéler, de rendre accessible et utilisable pour le plus grand nombre, en utilisant son fort potentiel pour améliorer l'image de la ville par des aménagements de qualité.

4 En France, plusieurs villes fluviales ou fluvio-maritimes, confrontées à la crise de leurs secteurs industriels, ont entamé des réflexions sur le devenir des espaces infrastructurels et industriels délaissés et fortement dégradés dès le début des années 1980, afin de réorganiser leurs installations portuaires et de trouver de nouvelles affectations aux emprises libérées. Les ports maritimes de Marseille, du Havre, de Saint-Nazaire et de Dunkerque ont pu constater les nombreux obstacles d'ordre juridique, financier et technique qu'implique une intervention sur ces emprises.

5 La redécouverte des espaces portuaires semble avant tout fournir une occasion pour insuffler une nouvelle dynamique aux villes qui investissent dans des sites en friche à 
proximité des centres urbains. La mise en valeur du patrimoine local, l'implantation de nouvelles activités, la création ou l'amélioration des logements, associées à des aménagements de qualité capables de redéfinir les espaces, contribuent à recentrer les villes sur leurs cœurs historiques et à transformer les traditionnelles activités d'échange et d'animation en nouvelles activités culturelles et ludiques.

Coupée de son port par la construction d'une base sous-marine lors de la Seconde Guerre mondiale, la ville de Saint-Nazaire voit émerger depuis plus d'une vingtaine d'années des projets visant à recréer un lien entre le centre-ville et le port dans le cadre plus vaste du réaménagement urbain de son front de l'océan (fig. $\left.\mathbf{n}^{\circ} \mathbf{1}\right)$. Avec les objectifs affirmés de renforcer les activités portuaires sur le site de Saint-Nazaire et de revitaliser le secteur par la mixité des fonctions urbaines, touristiques, culturelles et identitaires, le rapprochement ville-port s'est concrétisé par de nombreuses actions, par une série d'initiatives en matière de développement économique et par la multiplication d'opérations culturelles et de loisirs. La mise en scène ludique, associée à la mise en valeur du patrimoine local, demeure souvent le principe stratégique des opérations de régénération de cœur de ville.

\section{Figure 1}

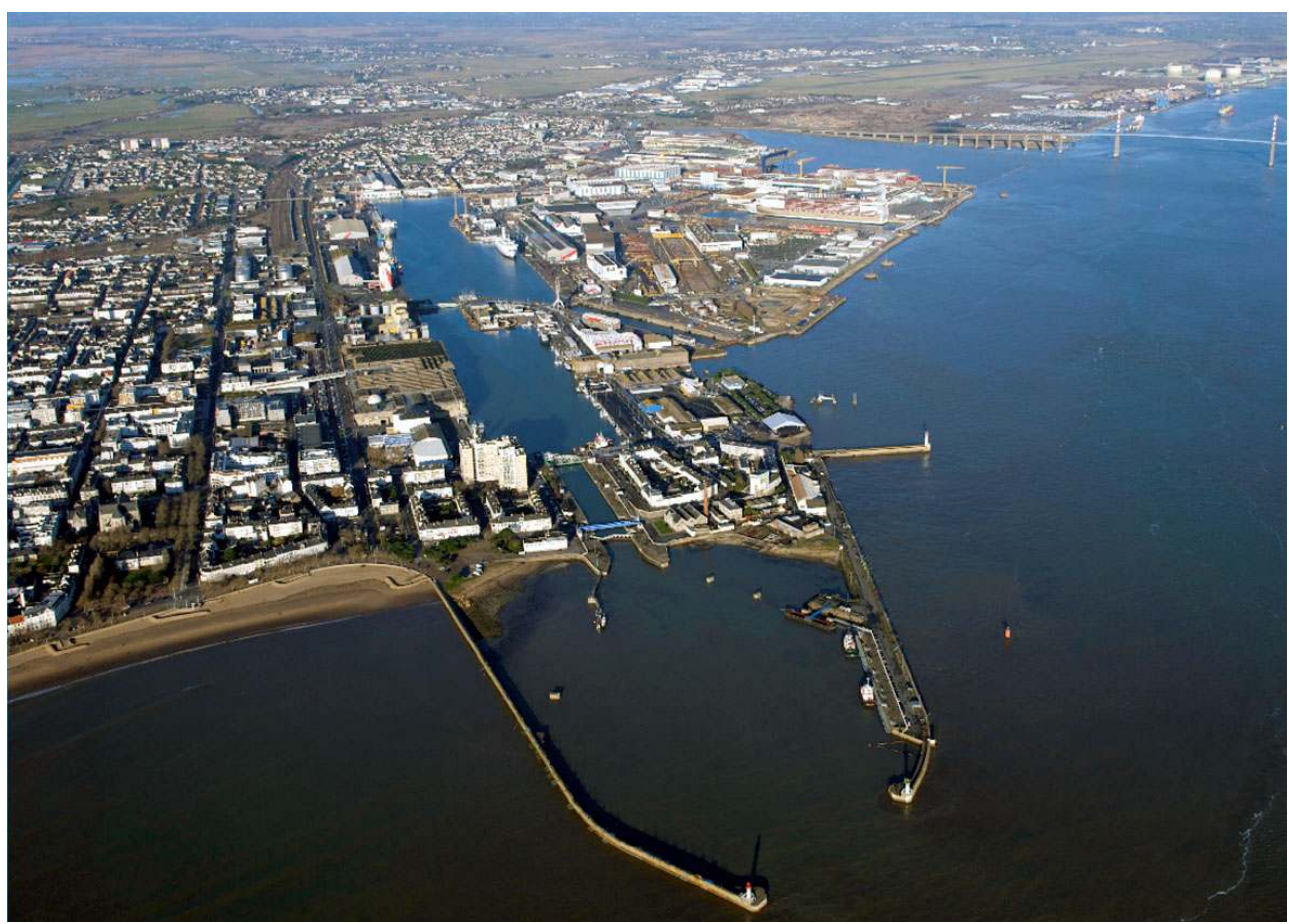

Vue aérienne de la ville de Saint-Nazaire avec les nouveaux aménagements autour de la base sousmarine. Extrait de éco.métropole Nantes Saint-Nazaire : construire la ville autour du fleuve, mars 2009. (c) SAMOA. Société d'Aménagement de la Métropole Ouest Atlantique.

7 La base sous-marine, qui a été labellisée « Patrimoine du $\mathrm{XX}^{\mathrm{e}}$ siècle » par le ministère de la Culture et de la Communication en 2010, est ainsi devenue emblématique d'une nouvelle approche qui considère la culture et le patrimoine des villes et des territoires sous toutes leurs formes, ne se limitant pas aux chefs d'œuvre et aux grands ouvrages. Ce sont également des biens ou des lieux plus ordinaires qui contribuent aussi à des cadres de vie exceptionnels. Comme le montre bien le cas de Saint-Nazaire, expérimenter une démarche de réinterprétation contemporaine du patrimoine militaire peut également 
susciter un sentiment d'appartenance et favorise une appropriation par les citoyens de l'espace qu'ils habitent ensemble, contribuant de même au renforcement des politiques d'agglomération.

Se référer explicitement à cette thématique et aux spécificités de cette ville moyenne française nous amène à concentrer notre analyse sur le projet urbain contemporain en tant qu'élément promoteur de mutations.

\section{Du bunker militaire à l'infrastructure culturelle}

Située sur l'Atlantique et à l'embouchure de la Loire, Saint-Nazaire devient au XIX siècle l'avant-port de Nantes. C'est à cette époque que la florissante activité des chantiers navals, destinée à la construction des transatlantiques, confirme la vocation de la ville à devenir un des principaux ports d'embarquement européen pour les traversées océaniques. À partir de 1862, les lignes transatlantiques pour l'Amérique centrale partent de Saint-Nazaire et les premiers chantiers navals s'implantent sur le site de Penhoët. La croissance de la ville, liée au commerce maritime et à la construction navale, sera caractérisée par la confluence des voies principales sur le bassin portuaire, au centre de l'ancienne ville, et par la disposition des plus importants édifices publics près de l'eau, réalisant ainsi un front de mer qui devient de référence pour la petite ville (fig. $\mathbf{n}^{\circ} \mathbf{2}$ ).

Figure 2

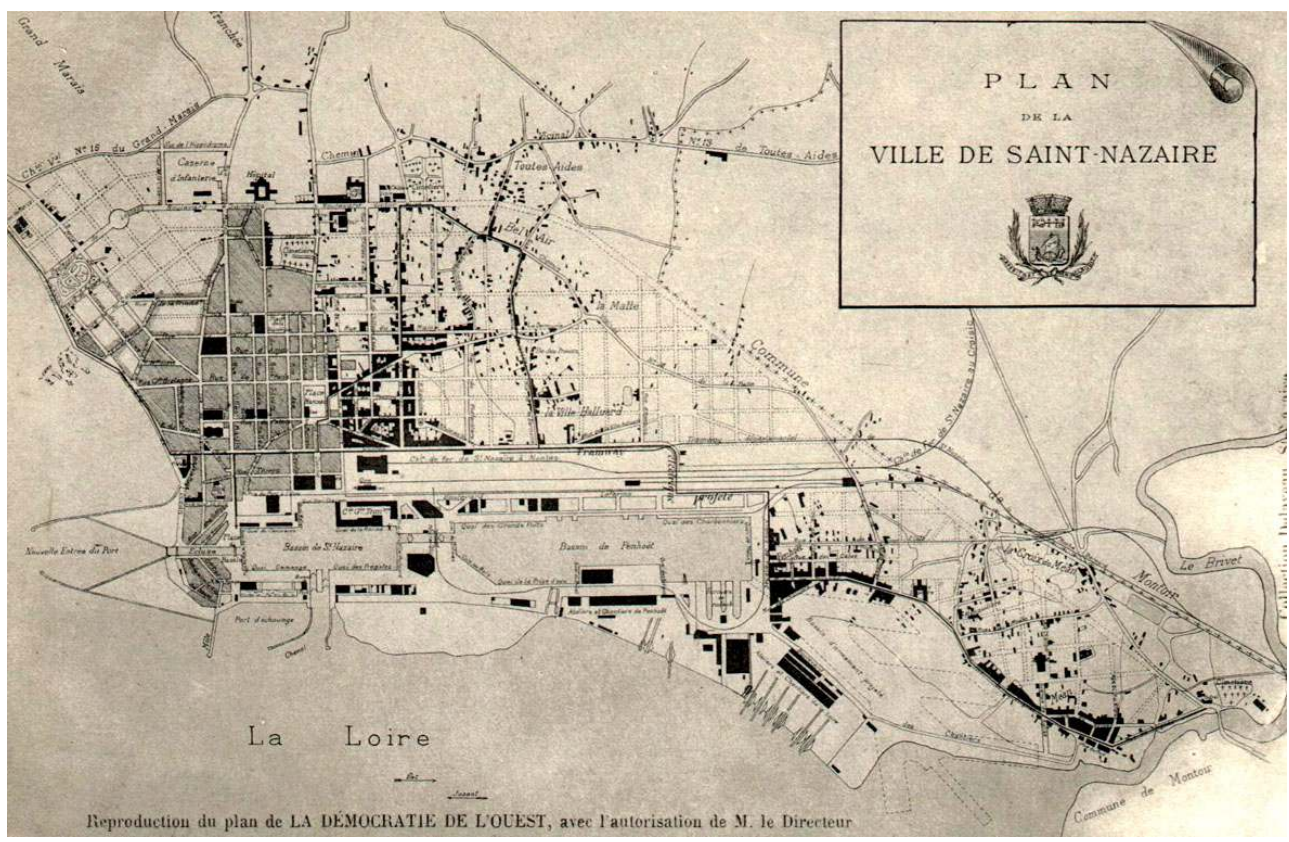

Plan de la ville de Saint-Nazaire, fin XIXe siècle.

(c) Collection particulière.

Pendant le second conflit mondial, les nazis intègrent le port de Saint-Nazaire au dispositif de surveillance du Mur de l'Atlantique ${ }^{2}$, confiant à l'Organisation Todt ${ }^{3}$ (OT) l'analyse du site pour le choix d'emplacement d'une nouvelle base sous-marine.

En charge de la réalisation d'un grand nombre de projets de construction, dans les domaines civil et militaire, tant en Allemagne, durant la période qui a précédé la guerre 
et pendant celle-ci, que dans les pays d'Europe sous domination nazie, l'OT était également engagée à l'époque dans la construction du Mur de l'Atlantique. Dans ce cadre, elle désigna une vaste zone du front de mer de Saint-Nazaire, près de son centre habité, pour construire une grande base sous-marine au bord du bassin portuaire, décrétant ainsi la démolition de l'ancienne gare transatlantique.

Construite en 1941, la base sera transformée en 1944 pour atteindre ses dimensions définitives ( $301 \mathrm{~m}$ de long, $130 \mathrm{~m}$ de large, $18 \mathrm{~m}$ de haut et $39000 \mathrm{~m}^{2}$ de superficie) et accueillir à l'intérieur de ses 14 alvéoles 20 sous-marins, une ligne ferroviaire traversante, des gares de pompage, des ateliers, des bureaux, des entrepôt de carburant, des logements ouvriers individuels et collectifs, une centrale électrique et des plates-formes de défense aérienne.

Danièle Voldman remarque que «l'édification de ces bases sous-marines était bien à proprement parler des travaux d'urbanisme qui ont durablement transformé le tissu des villes portuaires concernées $»^{4}$.

Contrairement aux villes de Lorient, Brest, Bordeaux, La Pallice-La Rochelle, dont les bases militaires étaient placées loin des centres habités, la base de Saint-Nazaire a donc la particularité de se situer le long d'un front de mer urbanisé, en faisant de cet emplacement et de son énorme façade aveugle les éléments matériels de la fracture entre la ville et l'eau, et restant pour l'avenir l'élément symbolique majeur de l'encombrant héritage historique de l'occupation nazie.

Cible principale des bombardements alliés, la base sous-marine a révélé sa capacité d'y résister grâce à la mise au point d'une structure type Fangrost, réalisée avec des blocs en béton armé de grande épaisseur superposés et posés sur le plancher de couverture de la base pour la protéger; cependant son emplacement a attiré les attaques aériennes sur le front de mer et le port, lieux qui seront détruits.

L'architecte Noël Le Maresquier, nommé architecte en chef de la reconstruction de SaintNazaire dès 1943, tiendra compte de la présence symbolique et matérielle de la base allemande dans l'élaboration de son plan, inspiré des principes de l'urbanisme moderne.

En réaffirmant le bouleversement du site portuaire déterminé lors de la réalisation de la base, ce plan réitère son incompatibilité avec le centre-ville, qui sera alors redessiné loin de l'eau.

L'axe majeur du centre-ville deviendra l'avenue de la République, parallèle à la darse mais loin de celle-ci, où seront installés les nouveaux îlots urbains à vocation résidentielle et commerciale.

19 Il faudra attendre les années 1980 pour voir apparaitre les premières études et projets de requalification urbaine axés sur la réconciliation entre la ville et le port. Dans ces mêmes années, la notion de monument, surtout dans ses relations au patrimoine ${ }^{5}$, connaît un certain nombre de mutations. Les débats dans lesquels s'engageront à cette époque de nombreux architectes et institutions concernant la tutelle de l'héritage construit laisseront émerger l'idée que les bâtiments puissent être considérés comme des monuments au-delà de leur statut et de leur usage d'origine.

20 À la même époque, une nouvelle approche de la conception architecturale est expérimentée. L'intérêt porté jusque-là à la typologie comme figure opératoire du projet s'affaiblit, et laisse émerger le potentiel de création de formes, correspondant à des édifices de grandes dimensions. Ces entités architecturales se réglant à la grande échelle, occuperaient une part conséquente de territoire, au point de constituer elles-mêmes des 
éléments territoriaux. Agencés par manipulation, insertion, hybridation, ces grands bâtiments seraient capables d'intégrer des programmes fort différenciés et d'absorber les éléments venant du terrain. Les grands bâtiments de guerre - et la base sous-marine de Saint-Nazaire parmi eux - figurent parmi les composants de cette famille architecturale. Bien que définis par Paul Virilio comme des « monuments funéraires du rêve allemand " ${ }^{6}$, ils ne témoignent pas des caractéristiques spécifiques des architectures monumentales au sens ancien du terme (usage public, identification aux valeurs positives de la collectivité, relation structurante avec l'espace publique attenant). C'est en raison de leur dimensions et de leur morphologie que l'architecture a pu produire un changement radical dans l'usage et dans l'image, modifiant ainsi le rôle de l'édifice, et permettant sa transmission, renouvelée, aux générations successives.

21 Suivant la volonté de la Ville de diversifier sa base économique en développant des fonctions tertiaires, notamment touristiques et culturelles, la base sous-marine s'est montrée apte à devenir une véritable infrastructure culturelle, lieu d'accueil pour un certain nombre d'équipements à l'échelle de la ville et de son territoire métropolitain. Avec ses aménagements, cet édifice représente l'équipement phare du projet, l'élément qui montre la volonté de changement d'image de la Ville et l'investissement de la municipalité dans une action de renouvellement urbain.

En parallèle, et plus notamment en ce qui concerne le réaménagement du territoire autour de Saint-Nazaire, on assiste à cette époque à l'inversion des orientations urbaines autour de l'eau, convoquée en thème fédérateur.

\section{Un détournement monumental}

23 À la convergence de ces enjeux on peut situer le complexe programme d'opérations urbaines et stratégiques entrepris par la municipalité de Saint-Nazaire à partir des années 1980, dans le cadre de la rénovation des relations du port à la ville.

Parmi celles-ci nous rappelons, par ordre chronologique, le Plan de référence, dessiné en 1983 pour réactiver le centre-ville, ainsi qu'un document d'encadrement à caractère stratégique, élaboré en 1991 pour unifier les cinq Plans d'Occupation des Sols déjà existants.

Des concours internationaux sur le site portuaire, lancés entre 1994 et 1996, viendront s'ajouter à ce cadre et verront confiée l'élaboration du projet Ville-Port à l'architecte espagnol Manuel de Solà-Morales. Le plan ainsi conçu s'est structuré autour de la spécificité environnementale et paysagère des infrastructures portuaires de SaintNazaire. La stratégie visait à réactiver l'image de la ville tout en transformant l'ancien abri militaire en moteur de la régénération urbaine (fig. $\mathbf{n}^{\circ} \mathbf{3}$ ). 


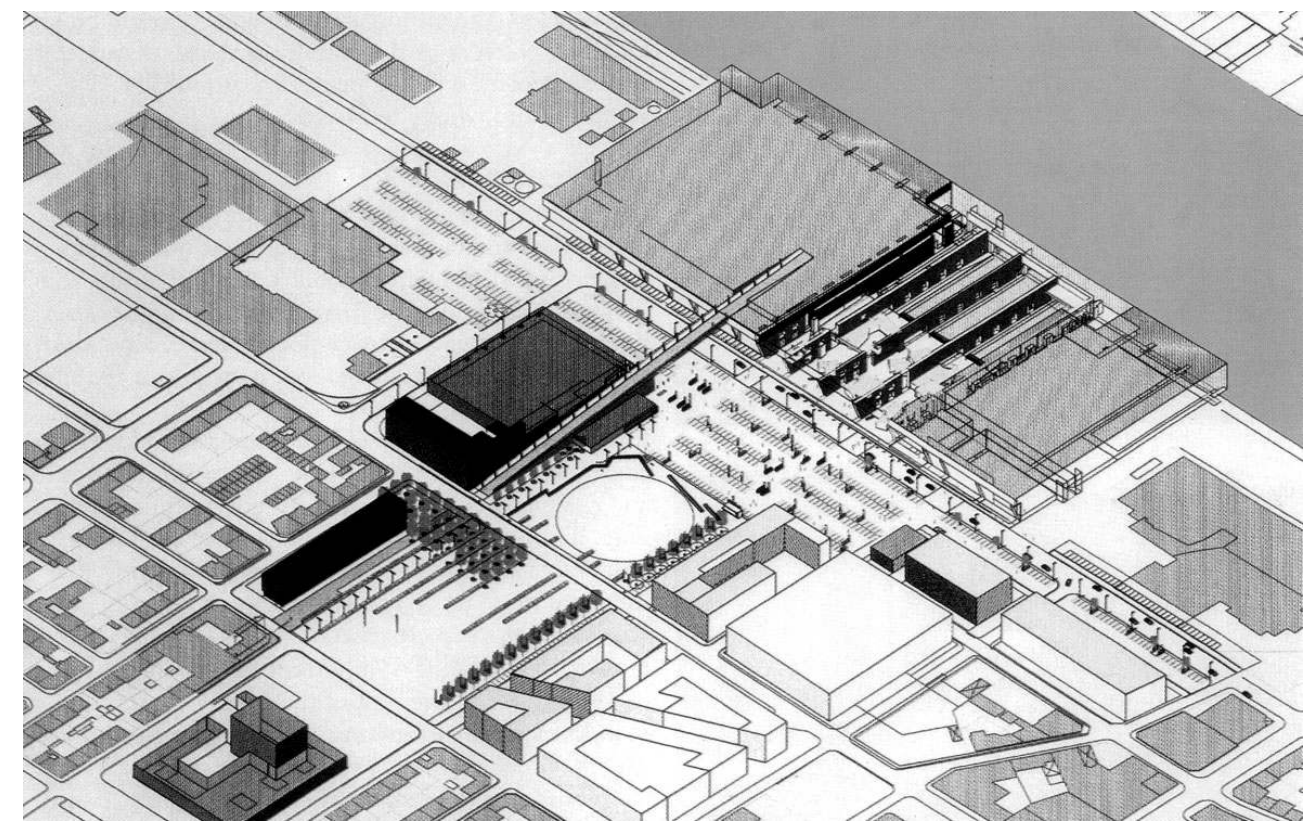

Axonométrie du projet Ville-Port, arch. Manuel de Solà-Morales. Une nouvelle orientation nord-sud de la ville est proposée avec l'aménagement de l'espace public sur le toit et face à la base sous-marine. Extrait de Solà-Morales M., mars 2000, City-Port Saint-Nazaire : the historical periphery, AQUAPOLIS, n ○I, p. 35.

En redessinant l'espace public autour de la base, le projet s'appuie sur l'intégration de deux principes fondamentaux. Le premier vise la reprise de la relation de la ville au port à travers la construction de nouveaux espaces publics entre le centre urbain et la darse du port (une rue piétonne, des nouveaux équipements). Le deuxième envisage de faire renouer des liens de fruition et d'identité entre la communauté et la mer, en reconnaissant un rôle moteur à la base sous-marine. À partir des réflexions évoquées, pourra s'affirmer une approche privilégiant l'ancien site militaire comme lieu d'événements culturels, souvent à caractère éphémère, qui fera de la base - et de l'espace public environnant - un monument, objectif majeur de la stratégie urbaine entreprise par la Ville.

Depuis 1998, le projet Ville-Port est entré dans la phase opérationnelle contribuant à définir un projet urbain et architectural de grande ampleur. L'étape principale de cette action a été la délimitation d'une zone prioritaire d'environ 15 ha et l'élaboration d'un projet de sauvegarde et de transformation de la base sous-marine. Le projet de SolàMorales contourne l'hypothèse de la démolition de la base, coûteuse et difficile à mener, en proposant l'édifice comme support sur lequel viendront s'implanter de nouvelles activités: office de tourisme, café-restaurant, boutique de souvenirs. Une des modifications principales de la base, l'ouverture de sa façade aveugle sur la ville, a été menée par la reconversion de quatre de ses alvéoles. La nouvelle surface, d'une étendue de $3500 \mathrm{~m}^{2}$, a été destinée à accueillir un nouvel équipement public: le musée de l'«Escale Atlantique », présentant une exposition thématique permanente sur l'histoire des chantiers navals et des transatlantiques. Afin de mieux souligner le changement d'orientation de la ville, qui regarde à nouveau vers l'eau, la deuxième modification majeure de l'ancien bâtiment a consisté en la construction d'une rampe qui conduit à un belvédère aménagé sur le toit de la base, ouvert sur la ville et sur le paysage portuaire. Le 
projet de Solà-Morales fait ainsi de la base sous-marine un lieu symbolique et stratégique, dont les principes pourront être intégrés par les opérations successives. De ce fait, les interventions conçues par Solà-Morales parviennent à opérer la désarticulation de l'ancien abri allemand, en introduisant une diversification entre alvéoles ouvertes et fermées qui accentue la flexibilité d'usages possibles pour l'édifice, ainsi que les modalités et les temporalités de sa fruition.

Cette opération, qui agit au niveau du corps physique de la base, la transforme en une structure permanente de grande échelle, prête à accueillir d'autres éléments, temporaires et de plus petite échelle, qui viendront s'y greffer.

Le mode grammatical de définition de ce projet rappelle évidemment celui des mégastructures. De même, concernant le programme, Solà-Morales affirme: "une densité diffuse de fonctions légèrement superposées, des limites pas trop déterminées où prévalent le vide et le niveau du sol, et une sensation d'espace excessif et peu défini sont à accepter et à apprécier, en étant, justement, le caractère du lieu $»^{7}$. Ces réflexions recoupent en plusieurs points certaines hypothèses d'il y a quarante ans, période où l'architecture commence à s'envisager "indéterminée et a-formelle", dans laquelle " chaque élément en vaut un autre ", posant les bases de l'indéfinition programmatique telle qu'elle est largement pratiquée dans l'architecture contemporaine.

De ce fait, les interventions qui ont suivi le projet de Solà-Morales tâchent d'assumer les indications implicites de son projet et d'en intégrer les principes.

Ainsi, des événements temporaires organisés près de la base ont contribué à construire un nouvel imaginaire portuaire, engendrant par là la définition d'un niveau symbolique et identitaire inattendu pour ce lieu, à l'origine des actions urbaines et architecturales successives.

Entre 1990 et 1991 l'éclairage nocturne du port, dénommé la Nuit des docks ${ }^{9}$, œuvre de l'artiste Yann Kersalé, inauguré lors des Fêtes de la mer (devenues les Escales), contribuera à forger un imaginaire qui encouragera la communauté à renouer des liens avec ce lieu, longtemps emblème de l'occupation allemande, puis de la crise des chantier navals.

En 2007, suite à un concours, le groupe d'architectes urbanistes LIN (Finn Geipel et Giulia Andi), a eu la charge de poursuivre les opérations de transformation de la base (fig. $\left.\mathbf{n}^{\circ} \mathbf{4}\right)$. L'intervention s'est axée sur la reconversion en rue intérieure de l'ancienne voie de chemin de fer jadis traversant la base pour l'acheminement des pièces mécaniques, ainsi que sur les alvéoles 13 et 14, dernières des 6 alvéoles à flot douées d'un bassin interne, aménagées pour accueillir deux équipements culturels : le LiFE (Lieu International des Formes Émergentes) et le VIP (Équipement des musiques actuelles). Le LiFE est un monoespace équipé d'une scénographie minimale. Se trouvant sur l'ancien plan d'eau, la salle peut s'ouvrir vers le bassin portuaire à l'aide d'une grande porte en accordéon. Le VIP occupe un des volumes « intérieurs » de la base. Celui-ci accueille une salle de 600 places, volume cubique et rudimentaire, complétée par un squelette métallique qui abrite un bar, des loges et le centre de documentation ${ }^{10}$. 
Figure 4

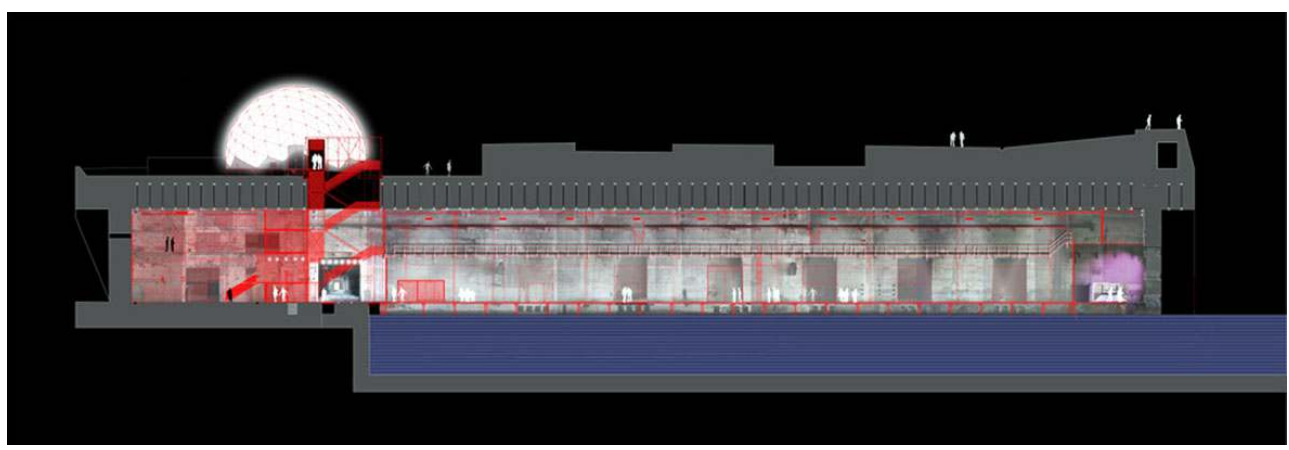

Coupe transversale de l'alvéole 14 de la base sous-marine avec les parcours verticaux de montée sur le toit. Extrait du dossier Alvéole 14 Transformation de la Base sous-marine Saint-Nazaire, 2007.

(c) Architectes urbanistes LIN Finn Geipel + Giulia Andi.

Il s'agit donc d'un projet qui s'appuie entièrement sur les traits constitutifs de l'ancienne base, eux-mêmes déjà soulignés par le projet de Solà-Morales : « la nature rudimentaire et intense de l'existant est prolongée par des installations élémentaires d'un autre ordre ${ }^{11}$.

C'est donc à l'aide de structures légères et provisoires en fer et verre que les grands espaces de plus de $1600 \mathrm{~m}^{2}$ voués à la création, à l'expérimentation et à la présentation de nouvelles formes artistiques et musicales contemporaines - avec un bar, un centre de documentation et un studio d'enregistrement et de production musicale - ont été aménagés. Superposés sur différents niveaux, les espaces communs sont connectés aux parcours verticaux qui amènent au pavillon belvédère en forme de dôme géodésique placé sur le toit.

36 Cette architecture interprétative, de reprise, de valorisation, et de détournement, a opéré une profonde mutation du rôle de la base allemande dans l'imaginaire collectif des habitants, en rénovant le rapport de la ville avec l'eau.

Depuis ces aménagements, Saint-Nazaire est devenue un lieu d'attraction touristique et culturelle. La base a été, par exemple, l'une des étapes du parcours artistique et festif de la deuxième édition de la biennale d'art contemporain Estuaire 2009. Dans ce cadre, à partir du 2009 et jusqu'en 2011, le paysagiste Gilles Clément a entrepris la réalisation de trois jardins publics à l'intérieur de la base (fig. $\mathbf{n}^{\circ}$ 5). Il s'agit d'un projet évolutif qui se montre comme un "lieu de résistance ", composé d'espèces appartenant à l'Estuaire et, pour partie, capable d'accueillir des espèces convergentes (orpins) compatibles avec le climat et le «non-sol » de la base. 


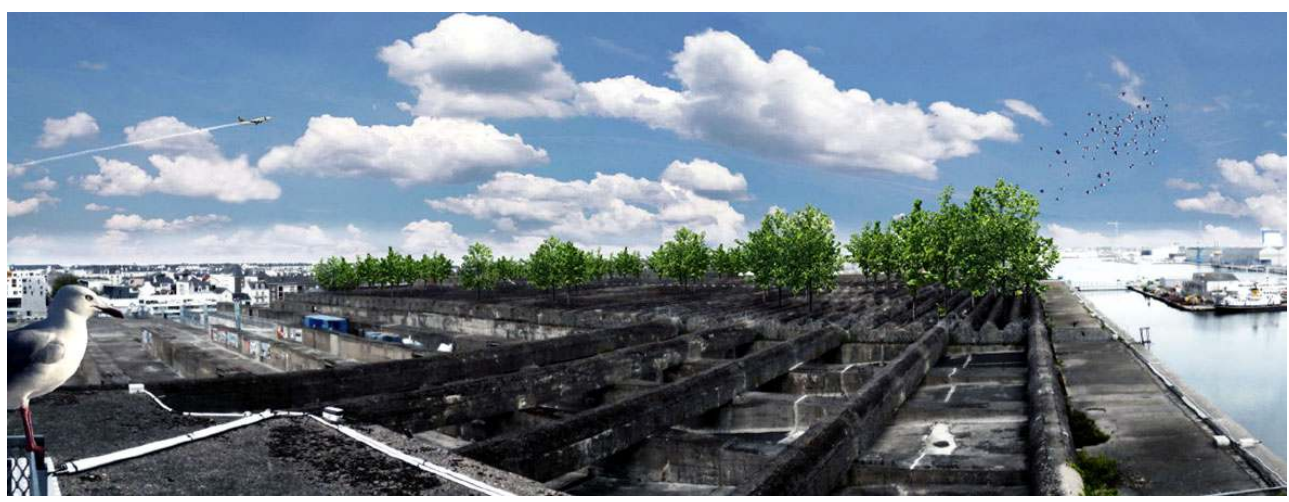

Vue du projet du Jardin du Tiers Paysage couvrant le toit de la base sous-marine, paysagiste Gilles Clément, 2009. Extrait du Jardin du Tiers Paysage, Toit de la Base sous-marine de Saint-Nazaire. Biennale d'art contemporain Estuaire 2009. Le lieu Unique. Nantes.

(c) Gilles Clément / Coloco.

38 Le premier jardin, dit du tiers paysage - en faisant écho à la plus récente recherche du paysagiste, focalisée sur «l'ensemble des lieux délaissés par l'homme ${ }^{12}$ - a prévu la plantation de 107 peupliers trembles entre les poutres de béton des chambres d'éclatement des bombes. Leur feuillage léger, toujours en mouvement par le vent, émerge sur le toit de la base, pour la transformer de façon permanente.

Une partie centrale, inachevée, de l'édifice, de part et d'autre d'une perspective constituée par les portes en enfilade, liant entre elles les chambres d'éclatement, accueillera en 2010 le Jardin des orpins et des graminées. La fosse rectangulaire située près du Dôme, enfin, sera le lieu de constitution spontané du troisième jardin, le Jardin des étiquettes, créé par les plantes qui y arriveront spontanément, par le vent ou les oiseaux, et qui seront identifiées et étiquetées au fur et à mesure de leur arrivée.

\section{Le pari d'un territoire métropolitain}

40 Pour mieux encadrer le sens des opérations visant la régénération urbaine de SaintNazaire, il est nécessaire d'en rappeler les similitudes avec le projet de la voisine Ile de Nantes, axé sur le réaménagement de ses territoires portuaires et industriels et sur le rôle stratégique attribué à l'espace public ${ }^{13}$. Parmi les objectifs communs aux deux sites, nous retrouvons la volonté de "faire la ville» et « de rouvrir la ville sur l'eau ». Alors que Saint-Nazaire confirme une transition qui l'a vue passer en vingt ans d'un statut de ville portuaire et industrielle en reconversion à celui de ville côtière à fort potentiel touristique, le rapprochement métropolitain à la ville de Nantes constitue un autre fait marquant de sa dynamique territoriale.

41 Le projet de développement d'une métropole associant les villes de Nantes et de SaintNazaire fait de la Loire, de son identité et de sa lisibilité, le fil conducteur de la recomposition de tout un territoire ${ }^{14}$. La ville de Saint-Nazaire a longtemps été l'avantport de Nantes. Elle s'est donc organisée et construite à partir, et autour, de cette fonction portuaire. Alors que le pôle nantais concentre les fonctions tertiaires et administratives, celui de Saint-Nazaire montre une forte spécialisation productive et résidentielle. Cette 
spécificité territoriale se pose comme un atout essentiel pour la métropole dont le développement apparaît à ce jour en équilibre.

Saint-Nazaire, dans son agglomération d'environ 150000 habitants, a connu pendant les dernières décennies un développement significatif dans plusieurs domaines: social, économique et urbain. Cette ville joue donc son rôle majeur sur le destin de la façade océane d'une métropole bipolaire.

Près de 800000 habitants vivent dans la métropole Nantes - Saint-Nazaire, cinquième de France par son poids démographique; si l'on y ajoute encore les aires voisines de La Baule et de son littoral, du vignoble au sud et d'Ancenis en amont, c'est presque un million de personnes qui vivent dans le périmètre de l'estuaire de la Loire.

Dans ce cadre, les projets Ville-Port et Ile de Nantes présentent de très nombreuses similitudes en ce qui concerne la stratégie des mutations des territoires historiquement liés par un destin industrialo-portuaire. Ces projets fortement dessinés et organisés accordent, parmi les points forts, une tension structurante de l'espace public, de la mixité des activités et des activités culturelles qui y sont conçues comme l'un des principaux vecteurs de réappropriation de l'espace.

Le projet Ville-Port constitue l'une des réponses apportées à la crise par la municipalité et répond à récupérer la séparation croissante entre la ville et le port. La convergence des enjeux urbains, industriels et portuaires, a permis une accélération de la mutation urbano-portuaire, concentrée sur les espaces proches de la base sous-marine allemande, ayant pour objectifs principaux le développement du projet ville-croisière, projet à la fois touristique et culturel. Même si les chaos de l'histoire ont détourné le centre-ville de son port, la ville revient vers la mer et s'engage, à la fin des années 1990, à relancer une dynamique sur le secteur de la base sous-marine, permettant une mutation des fonctions et une réappropriation de l'espace par la population nazairienne, au sein du Projet Global de Développement.

En faisant le pari de se réapproprier la base et l'espace public, de l'ouvrir à la fois sur le port et sur la ville, d'en faire un lieu pour les citoyens et les visiteurs, la ville de SaintNazaire, sous l'impulsion du maire Joël Batteux, a reconstitué une nouvelle identité portuaire à la ville par un pôle majeur d'attractivité. De manière analogue, parmi les principes fondateurs du projet de l'Ile de Nantes développé par le paysagiste Alexandre Chemetoff, nous retrouvons le plan évolutif et la mise en place d'un réseau d'espaces publics qui ouvrent le centre de l'île vers le fleuve, traité comme une entité géographique et historique à part entière. On observe, de plus, la forte prégnance des espaces publics servant de lien entre les différents équipements, d'espace d'accueil et de promenade pour les habitants. La priorité donnée au traitement des berges sollicite la réappropriation collective de tels espaces qui, une fois associés aux équipements, contribuent à dessiner la trame de la ville.

Ces deux grands projets urbains, au-delà de leurs spécificités, participent d'une volonté commune qui ambitionne de redonner une centralité à des lieux en déshérence à travers une démarche de développement durable et propose la mixité des usages et des populations dans des sites en transformation. Les identifiants communs aux deux projets urbains sont bien à la fois le retournement vers le fleuve ou son estuaire et le refus de la table rase. Le succès précurseur de Ville-Port a créé les conditions de sa poursuite de part et d'autre des bassins et vers l'océan, confirmant de même le futur positionnement 
fructueux de Saint-Nazaire dans l'aire d'influence de l'Arc atlantique, qui connaît par ailleurs une croissance démographique sans précédent.

Suite à des premières interventions en centre-ville datant des années 1980 (le projet du Centre République et notamment le bâtiment Le Paquebot, par l'architecte Claude Vasconi), et au projet Ville-Port, un troisième volet d'aménagements vise à relier la zone portuaire au plateau marchand situé à 500 mètres par un grand projet urbain dit VillePort 2. Il s'agit d'un programme de développement sur vingt ans, confié aux architectes italiens Bernardo Secchi et Paola Viganò, qui relance, au cœur du processus de régénération urbaine, la destination culturelle déjà réservée à la base sous-marine, pour l'étendre à l'ensemble des édifices environnants. Parmi leurs propositions, l'installation de la scène culturelle Le Fanal dans l'ancienne gare, qui retrouverait l'ancienne couverture vitrée ; le réaménagement de la Maison du Peuple, qui abrite les organisations syndicales; le déplacement de l'Écomusée sur le toit-terrasse de la base, et, dans le quartier du Petit-Maroc, la construction de 700 logements sur sept à huit niveaux, des boutiques, des bistrots, des ateliers, un mur habité sur deux niveaux face à l'usine élévatoire, un hangar tout en longueur destiné aux activités portuaires, venant épouser la jetée est de l'avant-port, avec un restaurant à sa proue, et, à l'horizon 2020, la création d'un centre d'art contemporain avançant sur l'eau près du Vieux Môle.

$\mathrm{Au}$ cours des vingt dernières années, la métropole Nantes - Saint-Nazaire s'est construite des lieux, temps et espaces de gouvernance, lui permettant d'énoncer aujourd'hui un projet stratégique de développement partagé : le projet d'une Éco-Métropole dont le Schéma de Cohérence Territoriale (SCOT), approuvé en 2007, dessine les grandes orientations. Ce schéma, rassemblant cinq intercommunalités, est indiscutablement un élément essentiel d'une stratégie partagée, ainsi qu'un outil dans la maîtrise de l'aménagement de l'espace de ce territoire. Ses objectifs de densification des constructions, de réutilisation de l'existant, de développement de la mixité sociale et de valorisation de l'environnement sont convergents avec les nouveaux programmes sur l'Ile de Nantes et l'évolution du projet Ville-Port. L'introduction des thématiques liées au développement durable et à la question climatique mettent le grand territoire en première ligne. Ainsi, comme le rappelle le dossier de candidature du projet pour l'ÉcoMétropole, la métropole Nantes - Saint-Nazaire constitue un des rares exemples en France de métropole à deux têtes, et plus généralement de l'organisation d'un développement partagé à l'échelle d'un grand territoire ${ }^{15}$.

\section{La force d'entraînement de la volonté politique}

La nouvelle politique urbaine commencée dans les années 1980 s'est élargie du centreville jusqu'au site portuaire. À l'hypothèse qui prévoyait l'extension du territoire de la ville, Saint-Nazaire a préféré le principe de la régénération urbaine, basé sur la construction de la ville sur elle-même à travers un programme de transformations capable de définir, à court, moyen et long terme, une nouvelle relation entre la cité et son port. L'intégration de la base sous-marine à la ville et le rapport avec l'eau ont été parmi les principes guides du projet Ville-Port.

51 Cette stratégie urbaine se caractérise par la qualité architecturale des opérations ponctuelles et par une participation financière publique majoritaire. Le consensus politique et économique réalisé autour de cette opération (avec un investissement de 70 millions d'euros pour la seule première phase) place les projets Ville-Port et Ile de 
Nantes parmi les grands projets métropolitains français en cours. Alors qu'une démarche patrimoniale est appliquée à la base sous-marine, la municipalité décide de promouvoir une véritable économie touristique et culturelle à Saint-Nazaire et, en 1997, crée la société anonyme d'économie mixte SIREN (Société d'initiatives de la région nazairienne), présidée par le même maire de la ville, qui regroupe au sein du conseil d'administration les partenaires du développement local. Sa mission consiste à réaliser l'ensemble des opérations en fonction de quatre objectifs prioritaires : diversifier l'économie et amplifier les retombées financières sur le commerce local; développer l'emploi; amplifier l'attractivité et la valorisation de l'image de la ville.

52 Forte du succès de la première tranche d'aménagements, la Ville en a lancé une seconde (Ville-Port 2), par laquelle pas moins de 18 opérations publiques et privées sont programmées d'ici à 2011, dont une artère commerciale de $18500 \mathrm{~m}^{2}$, un hôtel trois étoiles de 75 chambres, un théâtre et des logements. L'opération en cours à Saint-Nazaire montre bien l'opportunité d'une telle stratégie pour les villes moyennes et petites qui, à cause de trop faibles moyens économiques et urbains, peinent à se rénover.

$\mathrm{Au}$ cours des deux dernières décennies, Saint-Nazaire a relancé son développement urbain et économique sans se référer nécessairement aux modèles des grandes métropoles européennes. D'autres possibilités existent, mais la spécificité symbolique et la valorisation du patrimoine culturel ont permis à Saint-Nazaire de prouver que l'identité d'une ville pouvait activer sa croissance $^{16}$. Selon la réflexion d'Ariella Masboungi, l'expérience de Saint-Nazaire montre «que l'alternative à la fuite de toutes les dynamiques urbaines en périphérie est possible si les lieux centraux savent s'adapter à la demande sociale et être attractifs pour le marché, en jouant sur la qualité ; qu'une volonté politique claire, optimiste et déterminée a une réelle force d'entraînement; que la fatalité n'existe pas, à condition de savoir être soi-même, de ne pas imiter d'autres modèles ou d'autres destins et de ne pas craindre les résultats toujours incertains de l'action urbaine $»^{17}$.

\section{NOTES}

1. - L'activité de recherche en cours, au sein du Dipartimento di Storia e Progetto nell'Architettura (DISPA, Université de Palerme, Italie) et du Laboratoire Infrastructure Architecture et Territoire (ENSA Paris Malaquais), s'intéresse aux phénomènes de régénération urbaine et s'inscrit dans le courant des recherches sur la culture de la transformation de la ville contemporaine.

2. - Le Mur de l'Atlantique est l'objet de la recherche internationale The Atlantic Wall. Linear Museum, qui a vu la participation de chercheurs européens renommés autour de la proposition de réaliser un "musée linéaire" du Mur de l'Atlantique. Cette recherche a été publiée dans le catalogue et sur le site web de l'exposition réalisée en 2005 au Politecnico de Milan. Voir: PRELORENZO, Claude. Le mur de l'Atlantique dans la modernité, <http:// www.atlanticwall.polimi.it/> [date de consultation 21.01.09].

3. - Fondée par Fritz Todt et chargée par le nazisme de la construction d'œuvres civiles et militaires depuis 1933, l'organisation Todt comptait parmi ses travailleurs des salariés, des volontaires - techniciens civils et militaires - et des déportés, forcés, issus de différents groupes 
sociaux et ethniques. À la mort de Todt en 1942, Albert Speer sera chargé de la diriger. Voir : VIRILIO, Paul. Bunker archéologie, étude sur l'espace militaire européen de la Seconde Guerre mondiale. Paris : Édition Galilée, 2008, p. 38 (1édition 1975).

4. - Voir : VOLDMAN, Danièle. « Les villes françaises dans les deux conflits mondiaux 1914-1945 ", in PICON, Antoine (dir.). La ville et la guerre. Besançon: Les éditions de l'imprimeur, 1996, p. 198.

5. - Voir : ROUILLARD, Dominique. Architecture contemporaine et monuments historiques. Guides des réalisations en France depuis 1980. Paris : Éditions Le Moniteur, 2006.

6. - L'impact remarquable et profond des constructions du Mur de l'Atlantique a été démontré par le philosophe Paul Virilio à travers l'exposition Bunker Archéologie, présentée au musée des Arts Décoratifs à Paris (décembre 1975 - février 1976) et la publication du texte au titre homonyme. Voir : VIRILIO, Paul. Bunker archéologie, étude sur l'espace militaire européen de la Seconde Guerre mondiale. Paris : Édition Galilée, 2008, p. 40.

7. - Voir : de SOLÀ-MORALES, Manuel. "City-Port Saint-Nazaire : the Historical Periphery ", in Aquapolis, $\mathrm{n}^{\circ} \mathrm{I}$, mars 2000 , p. 32-37.

8. - Voir : BANHAM, Reyner. «A clip-on architecture ", in Architectural design, nov. 1965, p. 534 (tr. française : BANHAM, Reyner. «Une architecture d'attache », in Archigram, Paris, 1994).

9. - Les installations lumineuses réalisées en 1991 par l'artiste Yann Kersalé montrèrent un paysage nocturne inédit de la zone portuaire qui révèle le caractère extraordinaire de ses infrastructures et de ses bâtiments. Voir: MASBOUNGI, Ariella, de GRAVELAINE, Frédérique (dir.). Penser la ville par la lumière. Paris : Éditions de la Villette, 2003.

10. - Voir : LIN Geipel, Finn; ANDI, Giulia. Transformation de la Base sous-marine SaintNazaire, <http://www.pavillon-arsenal.com/img/conference/202/cp/PAV_202_CP.pdf > [date de consultation 21.01.09].

11. - Voir : LIN Geipel, Finn; ANDI, Giulia. Transformation de la Base sous-marine SaintNazaire, <http://www.pavillon-arsenal.com/img/conference/202/cp/PAV_202_CP.pdf > [date de consultation 21.01.09].

12. - Voir : CLÉMENT, Gilles. Manifeste du Tiers Paysage, 2004, <http://www.gillesclement.com/ fichiers/_tierspaypublications_92045_manifeste_du_tiers_paysage.pdf $>$ [date de consultation 21.01.09].

13. - Les installations lumineuses réalisées en 1991 par l'artiste Yann Kersalé montrèrent un paysage nocturne inédit de la zone portuaire qui révèle le caractère extraordinaire de ses infrastructures et de ses bâtiments. Voir: MASBOUNGI, Ariella, de GRAVELAINE, Frédérique (dir.). Penser la ville par la lumière. Paris : Éditions de la Villette, 2003.

14. - À ce propos, on peut lire le chapitre "La métropole : vers une stratégie du territoire ", in MASBOUNGI, Ariella (dir.), GRAVELAINE, Frédérique de. Nantes. La Loire dessine le projet. Paris : Éditions de la Villette, 2003, p. 179-191).

15. - La conférence 2008 Projets et gouvernances pour une écométropole a permis de démontrer que la métropole correspondait à une réalité vécue et qu'elle constituait une échelle pertinente pour la mise en œuvre de projets concrets d'une éco-métropole du XXI ${ }^{\mathrm{e}}$ siècle. Voir : http://www.nantessaintnazaire.fr/web/menu_les_conferences_metropolitaines.do).

16. - Voir : LECARDANE, Renzo. «Saint-Nazaire Ville-Port. Lo spazio pubblico della rigenerazione portuale / Saint-Nazaire Ville-Port. The public space of port regeneration ", in Portus, $\mathrm{n}^{\circ} 17$, mai 2009, p. 68-73.

17. - Voir : MASBOUNGI, Ariella. «Il processo di produzione in Europa », in Urbanistica, $n^{\circ} 126$, janvier-avril 2005, p. 15-19. 


\section{RÉSUMÉS}

$\mathrm{Au}$ cours des deux dernières décennies Saint-Nazaire a relancé son développement sans se référer nécessairement aux modèles des métropoles européennes. Concentrées autour de la zone portuaire, près de la base sous-marine allemande, les nouvelles opérations urbaines ont contribué à définir un important projet touristique et culturel. La spécificité symbolique et la valorisation du patrimoine militaire ont permis à Saint-Nazaire de montrer que l'identité d'une ville pouvait activer sa croissance.

Over the last two decades, the port city of Saint-Nazaire has encouraged urban redevelopment without necessarily following the models suggested by other European cities. An important cultural and tourist centre has been developed around the port zone, close to the German submarine base dating from the second World War. The symbolic specificity and the interpretation of this military heritage have shown, at Saint-Nazaire, how a town's special identity can foster growth.

\section{INDEX}

Keywords : Atlantic Wall, cultural infrastructure, military heritage, U-Boat bunker, urban regeneration, waterfront

Mots-clés : bunker U-Boat, infrastructure culturelle, patrimoine militaire, régénération urbaine, Saint-Nazaire

\section{AUTEURS}

\section{RENZO LECARDANE}

U. de Palerme/ DISPA et LIAT ENSA Paris-Malaquais) renzo.lecardane@unipa.it

\section{ZEILA TESORIERE}

U. de Palerme/ DISPA et LIAT ENSA Paris-Malaquais zeilatesoriere@unipa.it 